\title{
Improved total synthesis of incednam
}

\author{
Akira Takada, Kanjiro Uda, Takashi Ohtani, Shinya Tsukamoto, Daisuke Takahashi and Kazunobu Toshima \\ An alternative and improved total synthesis of incednam, the aglycon of the 24-membered macrolactam glycoside antibiotic \\ incednine, was accomplished. The synthesis was realized via construction of the 24-membered macrocycle using intramolecular \\ ring-closing olefin metathesis reaction as a key step. \\ The Journal of Antibiotics (2013) 66, 155-159; doi:10.1038/ja.2013.4; published online 6 February 2013
}

Keywords: incednam; incednine; olefin metathesis; total synthesis

\section{INTRODUCTION}

Incednam (1) is the aglycon of the 24-membered macrolactam glycoside antibiotic incednine (2), which was isolated by Imoto et al. ${ }^{1}$ in 2008 . Compound 2 exhibits significant inhibitory activity against the anti-apoptotic oncoproteins Bcl-2 and Bcl-xL, with a mode of action distinctly different from those of other agents that inhibit the binding capacity of $\mathrm{Bcl}-\mathrm{xL}$ to the pro-apoptotic protein Bax. Furthermore, these proteins are overexpressed in many cancer cells, resulting in the expansion of a transformed population and promotion of the multidrug-resistant stage. ${ }^{2-4}$ Therefore, 2 is expected to be a lead compound in the development of novel antitumor drugs. In addition, 2 is likely to be a useful tool for the further study of Bcl-2 and Bcl-xL functions. The identification of its target protein could provide insight into the anti-apoptotic mechanism of the Bcl-2 family proteins. From a chemical structural perspective, 1 and 2 contain unique features: an $\alpha$-methoxy- $\alpha, \beta$ unsaturated amide moiety and two independent conjugated polyene systems embedded in the 24 -membered macrolactam ring. Due to the nature of the highly conjugated polyene subunits, $\mathbf{1}$ and $\mathbf{2}$ are lightand acid-sensitive. Although $\mathbf{1}$ was also isolated from Streptomyces sp., ${ }^{1}$ its semi-synthesis from 2 has not been realized, in part due to its inherent chemical instabilities. However, their important biological activities and novel molecular architecture make $\mathbf{1}$ and $\mathbf{2}$ prime targets for chemical synthesis. The first total synthesis of $\mathbf{1}$ involved preparation of the $\mathrm{C} 1-\mathrm{C} 13$ subunit 3 and the $\mathrm{C} 14-\mathrm{C} 23$ subunit 4, and construction of the novel 24-membered macrocycle through Stille coupling between 3 and 4, followed by macrolactamization as shown in Figure 1.5 The present report describes an alternative and improved synthesis of 1 via construction of the 24-membered macrocycle by intramolecular ring-closing olefin metathesis reaction as a key step.

\section{RESULTS AND DISCUSSION}

The initial total synthesis of $\mathbf{1}$ was accomplished by preparation of the C14-C23 subunit 4, which could not be stored and was used immediately for the next step due to the instability. ${ }^{5}$ To circumvent this issue for the practical synthesis of $\mathbf{1}$, the retrosynthesis of $\mathbf{1}$ was redesigned via precursor $\mathbf{5}$ or $\mathbf{6}$ for intramolecular ring-closing olefin metathesis reaction ${ }^{6-8}$ to construct the 24-membered macrocycle concomitant with the labile C14-C21 tetraene unit at a later stage in the synthesis. The new retrosynthetic analysis of $\mathbf{1}$ is depicted in Figure 1. The convergent strategy applied to the construction of the 24-membered macrocycle is based on coupling of three domains: the $\mathrm{C} 1-\mathrm{C} 13$ subunit 3 containing the vinyl iodide moiety, ${ }^{5}$ the $\mathrm{C} 14-\mathrm{C} 18$ subunit 7 containing the vinyl stannane moiety, and the C19-C23 subunit $\mathbf{8}$ containing the amino group. This union was produced by application of intermolecular Stille coupling and amidation, followed by intramolecular ring-closing olefin metathesis reaction.

The synthesis of the triene subunit 7 , corresponding to the C14-C18 in 1, is summarized in Scheme 1. The known aldehyde $\mathbf{1 0}$ was prepared from ethyl 2-butynoate (9) in 5 steps by a procedure previously reported. ${ }^{9,10}$ Wittig reaction of 10 with $\mathrm{Ph}_{3} \mathrm{P}=\mathrm{CH}_{2}$ in $\mathrm{CH}_{2} \mathrm{Cl}_{2}$ provided the triene 7 in $88 \%$ yield. Next, the synthesis of the C19-C23 subunit 8 was accomplished starting from the alcohol 12, which was prepared as in the initial total synthesis of $1,{ }^{5}$ as shown in Scheme 2. The secondary alcohol 12 was converted into the mesylate 13 utilizing methanesulfonyl chloride $(\mathrm{MsCl})$ in pyridine $(\mathrm{Py})$, which was subsequently transformed into the azide $\mathbf{1 4}$ using $\mathrm{NaN}_{3}$ in $\mathrm{DMF}$ at $110{ }^{\circ} \mathrm{C}$ with stereochemical inversion in $88 \%$ overall yield. Deprotection of the tert-butyldiphenylsilyl (TBDPS) ether of $\mathbf{1 4}$ with tetrabutylammonium fluoride (TBAF) in THF, and subsequent oxidation of the resulting allyl alcohol 15 using $\mathrm{MnO}_{2}$ in $\mathrm{CH}_{2} \mathrm{Cl}_{2}$ provided the aldehyde $\mathbf{1 6}$ in $89 \%$ overall yield. Wittig reaction of $\mathbf{1 6}$ using $\mathrm{Ph}_{3} \mathrm{P}=\mathrm{CH}_{2}$ gave the diene 17 in $72 \%$ yield. Finally, reduction of the azide group of 17 using $\mathrm{PPh}_{3}$ under Staudinger's conditions ${ }^{11,12}$ furnished the amine $\mathbf{8}$ in $98 \%$ yield.

With the key fragments $\mathbf{7}$ and $\mathbf{8}$ in hand, attention turned to the total synthesis of $\mathbf{1}$ using 3 . Completion of the synthesis of $\mathbf{1}$ is 
<smiles>COC(=CC(C)=CC=CC=CC(OC(=O)O)C(C)(OC(F)(F)F)OC(F)(F)F)C(=O)O</smiles>

C1 - C13 subunit<smiles>CCCCC(C)=CC(C)=CC=CC(=CCC(C)C)PN</smiles>

4

C14 - C23 subunit

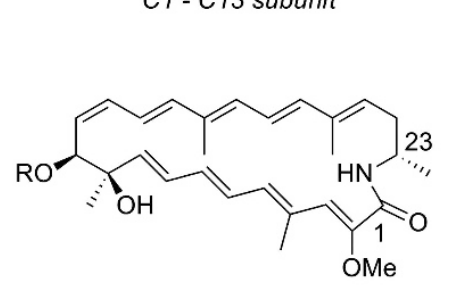<smiles>c1ccccc1</smiles>
Previous work: Ref. 5

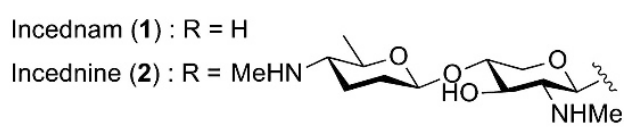
The present work

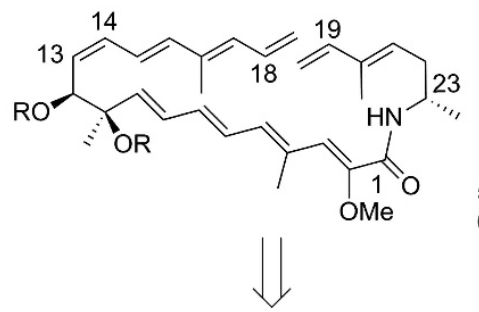<smiles>C=CC=CC(C=CC(CC)CC)=CC=C</smiles>

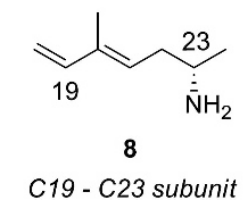

Figure 1 Retrosynthetic analysis of incednam (1).

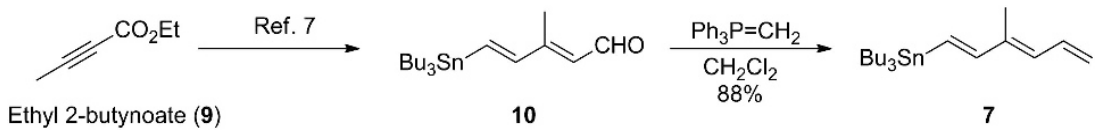

Scheme 1 Synthesis of the C14-C18 subunit 7.
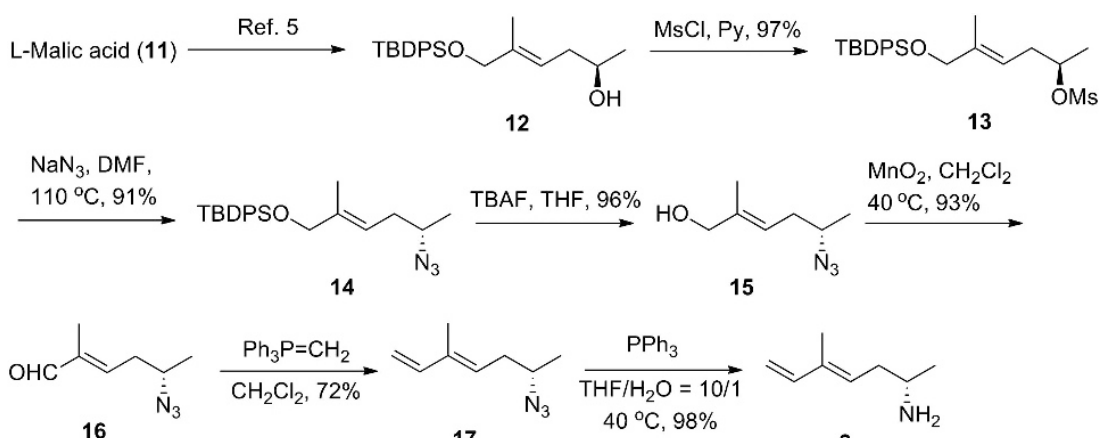
$\overline{\mathrm{N}}_{3}$

13

16
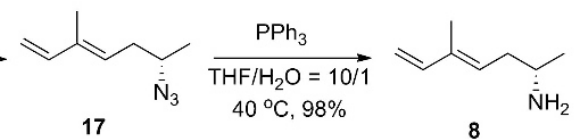

Scheme 2 Synthesis of the C19-C23 subunit 8.

summarized in Scheme 3. Amidation of $\mathbf{3}$ and $\mathbf{8}$ using 1-ethyl-3-(3dimethylaminopropyl)carbodiimide (EDC) and 4-dimethylaminopyridine (DMAP) in $\mathrm{CH}_{2} \mathrm{Cl}_{2}$ proceeded smoothly to give the amide 18 in $94 \%$ yield. Subsequently, Stille coupling of 18 and 7 using $\mathrm{Pd}_{2}(\mathrm{dba})_{3}$ in the presence of $\mathrm{LiCl}$ and $\mathrm{CuCl}$ in $\mathrm{DMF} / \mathrm{THF}^{13}$ gave the best result, providing the desired coupling product 19 in $71 \%$ yield. Removal of the triethylsilyl (TES) groups of 19 using TBAF and $\mathrm{AcOH}$ in THF furnished the diol 20 in $42 \%$ yield. Conditions for the intramolecular ring-closing olefin metathesis of $\mathbf{1 9}$ or $\mathbf{2 0}$ were rigorously explored using Grubbs first-generation, ${ }^{14}$ Grubbs second-generation, ${ }^{15}$ Hoveyda-Grubbs first-generation, ${ }^{16}$ Hoveyda-Grubbs second-generation, ${ }^{17}$ and Grela ${ }^{18,19}$ catalysts. Experimentation revealed that the best conditions were those using 19 and Grela catalyst 21 in the presence of $P$-methoxyphenol (PMPOH) and $\mathrm{MS} 3 \mathrm{~A}$ in $\mathrm{CH}_{2} \mathrm{Cl}_{2}$ to give 


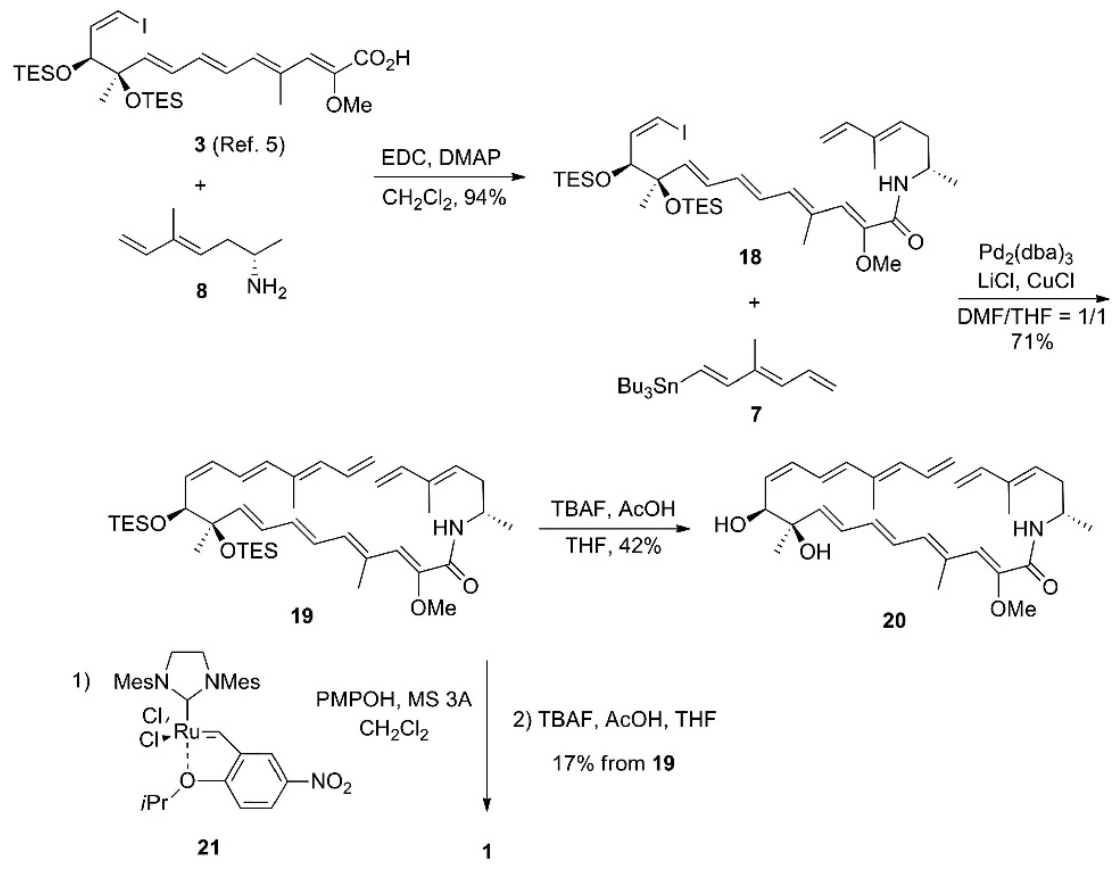

Scheme 3 Total synthesis of incednam (1).

incednam (1) in $17 \%$ overall yield after deprotection of the TES groups in the resulting cyclic product. ${ }^{1} \mathrm{H}-\mathrm{NMR},{ }^{13} \mathrm{C}-\mathrm{NMR}$, HRMS (ESI-TOF), and optical rotation data obtained for a sample of the synthetic incednam matched those of an authentic sample ${ }^{1}$ and of a sample from the initial synthesis of $1 .^{5}$

In conclusion, a novel convergent synthetic route was developed for incednam (1), which is the aglycon of the 24-membered macrolactam glycoside antibiotic incednine (2), using intramolecular ring-closing olefin metathesis reaction as a key step. Although the yield of the intramolecular ring-closing olefin metathesis reaction was not extremely high, the present synthetic route avoids the use of unstable fragments, such as $\mathbf{4}$, in the total synthesis of $\mathbf{1}$. Furthermore, this approach shows potential for intramolecular ring-closing olefin metathesis even in a complex structure possessing polyene units. Additional studies related to the total synthesis of incednine (2) from 1 are currently underway. ${ }^{20}$

With great respect, we dedicate this work to Professor Kuniaki Tatsuta as a memorial to his total synthesis of 101 antibiotics. This research was supported in part by the MEXT-supported Program for the Strategic Research Foundation at Private Universities, 2012-2016, Scientific Research on Innovative Areas 'Chemical Biology of Natural Products' and JSPS Fellow 22.5820 from MEXT.

\section{EXPERIMENTAL PROCEDURE}

Melting points were determined on a micro hot-stage (Yanako MP-S3) and were uncorrected. Optical rotations were measured on a JASCO P-2200 polarimeter. ${ }^{1} \mathrm{H}-\mathrm{NMR}$ spectra were recorded on a JEOL ECA-500 $(500 \mathrm{MHz})$ spectrometer. ${ }^{13} \mathrm{C}$-NMR spectra were taken on a JEOL ECA-500 $(125 \mathrm{MHz})$ spectrometer in $\mathrm{CDCl}_{3}$ at room temperature, unless otherwise noted. ${ }^{1} \mathrm{H}-\mathrm{NMR}$ data were reported as follows: chemical shift in parts par million (p.p.m.)

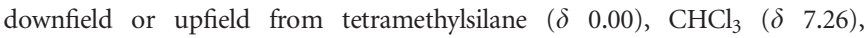
integration, multiplicity $(\mathrm{br}=$ broad, $\mathrm{s}=$ singlet, $\mathrm{d}=$ doublet, $\mathrm{t}=$ triplet, $\mathrm{q}=$ quartet, $\mathrm{m}=$ multiplet) and coupling constants $(\mathrm{Hz}) \cdot{ }^{13} \mathrm{C}$ chemical shifts were reported in p.p.m. downfield or upfield from $\mathrm{CDCl}_{3}\left(\delta\right.$ 77.36) or acetone- $d_{6}(\delta$ 30.60). ESI-TOF Mass spectra and APCI-TOF Mass spectra were measured on a Waters LCT premier XE. Silica-gel TLC and column chromatography were performed on Merck TLC 60F-254 (0.25 mm) and Kanto Chemical Co., Inc. (Tokyo, Japan) Silica-Gel $60 \mathrm{~N}$ (spherical, neutral), respectively. Air- and/or moisture-sensitive reactions were carried out under an atmosphere of argon using oven-dried glassware. In general, organic solvents were purified and dried using an appropriate procedure, and evaporation and concentration were carried out under reduced pressure below $30^{\circ} \mathrm{C}$, unless otherwise noted.

\section{(1E,3E,5E)-3-Methylhex-1,3,5-trien-1-tributylstannane (7)}

To a solution of $10(56.6 \mathrm{mg}, 146 \mu \mathrm{mol})$ in dry $\mathrm{CH}_{2} \mathrm{Cl}_{2}(1.12 \mathrm{ml})$ was added $\mathrm{Ph}_{3} \mathrm{P}=\mathrm{CH}_{2}(202 \mathrm{mg}, 731 \mu \mathrm{mol})$ under Ar atmosphere at room temperature. The reaction mixture was stirred for $15 \mathrm{~h}$ at the temperature, the mixture was quenched by addition of a saturated $\mathrm{NH}_{4} \mathrm{Cl}$ aq. $(1 \mathrm{ml})$. The resulting mixture was extracted with $\mathrm{CHCl}_{3}(2 \mathrm{ml} \times 3)$. The combined organic layer was washed with brine $(1 \mathrm{ml})$, dried over anhydrous $\mathrm{Na}_{2} \mathrm{SO}_{4}$, and concentrated in vacuo. Purification of the residue by column chromatography (hexane) on aluminum oxide activated, basic, Brockmann I gave 7 ( $49.0 \mathrm{mg}, 128 \mu \mathrm{mol}, 88 \%$ yield). Pale yellow syrup; $R_{f} 0.72$ (10/1 hexane/EtOAc); ${ }^{1} \mathrm{H}-\mathrm{NMR}\left(\mathrm{CDCl}_{3}\right.$, TMS) $\delta$ $6.71(1 \mathrm{H}, \mathrm{ddd}, J=10.0,11.2,16.9 \mathrm{~Hz}), 6.57(1 \mathrm{H}, \mathrm{d}, J=19.2 \mathrm{~Hz}), 6.30(1 \mathrm{H}, \mathrm{d}$, $J=19.2 \mathrm{~Hz}), 6.06(1 \mathrm{H}, \mathrm{d}, J=11.2 \mathrm{~Hz}), 5.26(1 \mathrm{H}, \mathrm{d}, J=16.9 \mathrm{~Hz}), 5.13(1 \mathrm{H}, \mathrm{d}$, $J=10.0 \mathrm{~Hz}), 1.87(3 \mathrm{H}, \mathrm{s}), 1.50(6 \mathrm{H}, \mathrm{m}), 1.31(6 \mathrm{H}, \mathrm{m}), 0.90(15 \mathrm{H}, \mathrm{m}) ;{ }^{13} \mathrm{C}-$ $\mathrm{NMR}\left(\mathrm{CDCl}_{3}\right) \delta 150.7,137.2,133.6,131.4,128.0,117.8,29.3,27.5,13.9,12.2$, 9.7; HRMS (ESI-TOF) $\mathrm{m} / z 385.19$ (385.1921 calcd for $\mathrm{C}_{19} \mathrm{H}_{37} \mathrm{Sn},[\mathrm{M}+\mathrm{Na}]^{+}$).

\section{(2E,5R)-1-tert-Butyldiphenylsilyloxy-5-methanesulfonyloxy-} 2-methylhex-2-ene (13)

To a solution of $12(1.30 \mathrm{~g}, 3.53 \mathrm{mmol})$ in dry pyridine $(19.5 \mathrm{ml})$ was added $\mathrm{MsCl}(410 \mu \mathrm{l}, 5.30 \mathrm{mmol})$ under $\mathrm{Ar}$ atmosphere at $0{ }^{\circ} \mathrm{C}$. After the mixture was stirred for $3 \mathrm{~h}$ at room temperature, the reaction was quenched by addition of water $(20 \mathrm{ml})$. The resulting mixture was extracted with EtOAc $(10 \mathrm{ml} \times 3)$. The combined organic layer was washed with brine $(20 \mathrm{ml})$, dried over anhydrous $\mathrm{Na}_{2} \mathrm{SO}_{4}$, and concentrated in vacuo. Purification of the residue by silica-gel column chromatography (hexane/EtOAc $=4 / 1)$ gave $13(1.53 \mathrm{~g}$, $3.42 \mathrm{mmol}, 97 \%$ yield). Colorless syrup; $R_{f} 0.60\left(2 / 1\right.$ hexane/EtOAc); $[\alpha]_{D}^{26}$ $+2.4^{\circ}\left(c \quad 0.28, \mathrm{CHCl}_{3}\right) ;{ }^{1} \mathrm{H}-\mathrm{NMR}\left(\mathrm{CDCl}_{3}, \mathrm{TMS}\right) \delta 7.65(4 \mathrm{H}, \mathrm{dd}, J=1.5$, $7.7 \mathrm{~Hz}), 7.39(6 \mathrm{H}, \mathrm{m}), 5.51(1 \mathrm{H}, \mathrm{dt}, J=1.7,7.5 \mathrm{~Hz}), 4.79(1 \mathrm{H}, \mathrm{ddq}, J=6.3,6.3$, $6.6 \mathrm{~Hz}), 4.06(2 \mathrm{H}, \mathrm{s}), 2.94(3 \mathrm{H}, \mathrm{s}), 2.52(1 \mathrm{H}, \mathrm{ddd}, J=6.6,7.5,14.0 \mathrm{~Hz}), 2.38$ $(1 \mathrm{H}, \mathrm{ddd}, J=6.3,7.5,14.0 \mathrm{~Hz}), 1.61(3 \mathrm{H}, \mathrm{s}), 1.41(3 \mathrm{H}, \mathrm{d}, J=6.3 \mathrm{~Hz}) 1.06$ 
$(9 \mathrm{H}, \mathrm{s}) ;{ }^{13} \mathrm{C}-\mathrm{NMR}\left(\mathrm{CDCl}_{3}\right) \delta 138.2,135.6,133.7,129.8,127.8,117.4,80.0$, 68.4, 38.6, 34.8, 26.9, 21.1, 19.4, 13.9; Anal. calcd for $\mathrm{C}_{24} \mathrm{H}_{34} \mathrm{O}_{4} \mathrm{SSi}$ : C, 64.53; H, 7.67; S, 7.18. Found: C, 64.37; H, 7.85; S, 7.46.

\section{(2E,5S)-5-Azido-1-tert-butyldiphenylsilyloxy-2-methylhex-2-ene} (14)

To a solution of $13(1.53 \mathrm{~g}, 3.41 \mathrm{mmol})$ in dry DMF $(20.0 \mathrm{ml})$ was added $\mathrm{NaN}_{3}$ (333 mg, $5.12 \mathrm{mmol}$ ) under $\mathrm{Ar}$ atmosphere at room temperature. After the mixture was stirred for $1.5 \mathrm{~h}$ at $110^{\circ} \mathrm{C}$, the reaction was quenched by addition of water $(20 \mathrm{ml})$. The resulting mixture was extracted with hexane/EtOAc $=1 / 1$ $(10 \mathrm{ml} \times 3)$. The combined organic layer was washed with brine $(20 \mathrm{ml})$, dried over anhydrous $\mathrm{Na}_{2} \mathrm{SO}_{4}$, and concentrated in vacuo. Purification of the residue by silica-gel column chromatography (hexane/EtOAc $=20 / 1$ ) gave 14 $\left(1.22 \mathrm{~g}, 3.10 \mathrm{mmol}, 91 \%\right.$ yield). Colorless syrup; $R_{f} 0.61$ (5/1 hexane/EtOAc); $[\alpha]_{\mathrm{D}}^{26}+6.6^{\circ}\left(c 0.28, \mathrm{CHCl}_{3}\right) ;{ }^{1} \mathrm{H}-\mathrm{NMR}\left(\mathrm{CDCl}_{3}\right.$, TMS) $\delta 7.67(4 \mathrm{H}, \mathrm{d}, J=6.6$ $\mathrm{Hz}), 7.39(6 \mathrm{H}, \mathrm{m}), 5.50(1 \mathrm{H}, \mathrm{t}, J=7.5 \mathrm{~Hz}), 4.07(2 \mathrm{H}, \mathrm{s}), 3.48(1 \mathrm{H}, \mathrm{tq}, J=6.6$, $6.9 \mathrm{~Hz}), 2.31(1 \mathrm{H}, \mathrm{ddd}, J=6.9,7.5,14.1 \mathrm{~Hz}), 2.22(1 \mathrm{H}, \mathrm{ddd}, J=6.9,7.5$, $14.1 \mathrm{~Hz}), 1.61(3 \mathrm{H}, \mathrm{s}), 1.23(3 \mathrm{H}, \mathrm{d}, J=6.6 \mathrm{~Hz}), 1.07(9 \mathrm{H}, \mathrm{s}) ;{ }^{13} \mathrm{C}-\mathrm{NMR}$ $\left(\mathrm{CDCl}_{3}\right) \delta 137.3,135.6,133.9,129.7,127.7,119.1,77.4,77.1,76.9,68.7,58.0$, 34.3, 26.9, 19.4, 19.2, 13.8; Anal. Calcd for $\mathrm{C}_{23} \mathrm{H}_{31} \mathrm{~N}_{3}$ OSi: C, 70.18; H, 7.94 . Found: C, 69.85; H, 8.00.

\section{(2E,5S)-5-Azido-2-methylhex-2-en-1-ol (15)}

To a solution of $14(1.91 \mathrm{~g}, 4.85 \mathrm{mmol})$ in dry THF $(38.0 \mathrm{ml})$ was added $1.0 \mathrm{M}$ TBAF in THF $(7.27 \mathrm{ml}, 7.27 \mathrm{mmol})$ under Ar atmosphere at $0{ }^{\circ} \mathrm{C}$. After the mixture was stirred for $3 \mathrm{~h}$ at room temperature, the reaction was quenched by addition of $\mathrm{H}_{2} \mathrm{O}(10 \mathrm{ml})$. The resulting mixture was extracted with EtOAc $(20 \mathrm{ml} \times 3)$. The combined organic layer was washed with brine $(10 \mathrm{ml})$, dried over anhydrous $\mathrm{Na}_{2} \mathrm{SO}_{4}$, and concentrated in vacuo. Purification of the residue by silica-gel column chromatography (hexane/EtOAc $=2 / 1,1 \% \mathrm{Et}_{3} \mathrm{~N}$ ) gave 15 (726 mg, $4.68 \mathrm{mmol}$, 96\% yield). Colorless syrup; $R_{f} 0.18$ (5/1 hexane/EtOAc); $[\alpha]_{\mathrm{D}}^{25}+11.9^{\circ}\left(\right.$ c $\left.0.49, \mathrm{CHCl}_{3}\right) ;{ }^{1} \mathrm{H}-\mathrm{NMR}\left(\mathrm{CDCl}_{3}, \mathrm{TMS}\right) \delta 5.45(1 \mathrm{H}, \mathrm{dt}, J=1.6$, $7.2 \mathrm{~Hz}), 4.03(2 \mathrm{H}, \mathrm{s}), 3.51(1 \mathrm{H}, \mathrm{m}), 2.27(2 \mathrm{H}, \mathrm{m}), 1.69(3 \mathrm{H}, \mathrm{s}), 1.26(3 \mathrm{H}, \mathrm{d}$, $J=6.6 \mathrm{~Hz}) ;{ }^{13} \mathrm{C}-\mathrm{NMR}\left(\mathrm{CDCl}_{3}\right) \delta 138.1,120.7,68.5,34.5,19.3,14.0$; HRMS (ESI-TOF) $m / z 128.1077\left(128.1075\right.$ calcd for $\mathrm{C}_{7} \mathrm{H}_{14} \mathrm{NO},\left[\mathrm{MH}-\mathrm{N}_{2}\right]^{+}$).

\section{(2E,5S)-5-Azido-2-methylhex-2-en-1-al (16)}

To a solution of $15(726 \mathrm{mg}, 4.68 \mathrm{mmol})$ in dry $\mathrm{CH}_{2} \mathrm{Cl}_{2}(46.8 \mathrm{ml})$ was added $\mathrm{MnO}_{2}$ (4.07g, $46.8 \mathrm{mmol}$ ) under $\mathrm{Ar}$ atmosphere at the room temperature. After the mixture was stirred for $15 \mathrm{~h}$ at $40{ }^{\circ} \mathrm{C}$, the mixture was filtered through a pad of Celite. The combined filtrates were concentrated in vacuo. Purification of the residue by silica-gel column chromatography (hexane/EtOAc $=2 / 1$ ) gave 16 (663 mg, $4.33 \mathrm{mmol}, 93 \%$ yield). Colorless syrup; $R_{f} 0.33$ (5/1 hexane/ EtOAc); $[\alpha]_{\mathrm{D}}^{24}+24.3^{\circ}\left(\right.$ c $\left.0.68, \mathrm{CHCl}_{3}\right) ;{ }^{1} \mathrm{H}-\mathrm{NMR}\left(\mathrm{CDCl}_{3}\right.$, TMS $) \delta 9.45(1 \mathrm{H}, \mathrm{s})$, $6.51(1 \mathrm{H}, \mathrm{dt}, J=1.4,7.2 \mathrm{~Hz}), 3.71(1 \mathrm{H}, \mathrm{m}), 2.55(2 \mathrm{H}, \mathrm{dd}, J=6.6,7.0 \mathrm{~Hz}), 1.78$ $(3 \mathrm{H}, \mathrm{s}), 1.35(3 \mathrm{H}, \mathrm{d}, J=6.6 \mathrm{~Hz}) ;{ }^{13} \mathrm{C}-\mathrm{NMR}\left(\mathrm{CDCl}_{3}\right) \delta 194.9,148.5,141.5,56.8$, 35.5, 19.5, 9.6; HRMS (ESI-TOF) $m / z 126.0917$ (126.0919 calcd for $\mathrm{C}_{7} \mathrm{H}_{12} \mathrm{NO}$, $\left.\left[\mathrm{MH}-\mathrm{N}_{2}\right]^{+}\right)$.

\section{(2E,4E,6E)-2-Azido-5-methylhepta-4,6-diene (17)}

To a solution of $16(175 \mathrm{mg}, 1.14 \mathrm{mmol})$ in dry $\mathrm{Et}_{2} \mathrm{O}(11.4 \mathrm{ml})$ was added $\mathrm{Ph}_{3} \mathrm{P}=\mathrm{CH}_{2}(450 \mathrm{mg}, 1.60 \mathrm{mmol})$ under Ar atmosphere at $0{ }^{\circ} \mathrm{C}$. The reaction mixture was stirred for $1 \mathrm{~h}$ at the room temperature, the mixture was quenched by addition of a saturated $\mathrm{NH}_{4} \mathrm{Cl}$ aq. $(5 \mathrm{ml})$. The resulting mixture was extracted with $\mathrm{Et}_{2} \mathrm{O}(5 \mathrm{ml} \times 3)$. The combined organic layer was washed with brine $(5 \mathrm{ml})$, dried over anhydrous $\mathrm{Na}_{2} \mathrm{SO}_{4}$, and concentrated in vacuo. Purification of the residue by silica-gel column chromatography (hexane/ $\left.\mathrm{Et}_{2} \mathrm{O}=30 / 1,1 \% \mathrm{Et}_{3} \mathrm{~N}\right)$ gave 17 ( $124 \mathrm{mg}, 822 \mu \mathrm{mol}, 72 \%$ yield). Pale yellow syrup; $R_{f} 0.71$ (10/1 hexane/EtOAc); $[\alpha]^{25}+12.1^{\circ}\left(c 0.52, \mathrm{CHCl}_{3}\right) ;{ }^{1} \mathrm{H}-\mathrm{NMR}$ $\left(\mathrm{CDCl}_{3}, \mathrm{TMS}\right) \delta 6.38(1 \mathrm{H}, \mathrm{dd}, J=10.6,17.5 \mathrm{~Hz}), 5.48(1 \mathrm{H}, \mathrm{t}, J=7.2 \mathrm{~Hz}), 5.14$ $(1 \mathrm{H}, \mathrm{d}, J=17.5 \mathrm{~Hz}), 4.99(1 \mathrm{H}, \mathrm{d}, J=10.6 \mathrm{~Hz}), 3.52(1 \mathrm{H}, \mathrm{m}), 2.35(2 \mathrm{H}, \mathrm{m})$, $1.77(3 \mathrm{H}, \mathrm{s}), 1.26(3 \mathrm{H}, \mathrm{d}, J=6.6 \mathrm{~Hz}) ;{ }^{13} \mathrm{C}-\mathrm{NMR}\left(\mathrm{CDCl}_{3}\right) \delta 141.2,136.8,127.5$, 111.9, 57.9, 35.0, 19.3, 12.1; HRMS (ESI-TOF) $\mathrm{m} / \mathrm{z} 124.1127$ (124.1126 calcd for $\mathrm{C}_{8} \mathrm{H}_{14} \mathrm{~N},\left[\mathrm{MH}-\mathrm{N}_{2}\right]^{+}$).

\section{(2E,4E,6E)-2-Amino-5-methylhepta-4,6-diene (8)}

To a solution of $17(124 \mathrm{mg}, 822 \mu \mathrm{mol})$ in $\mathrm{THF} / \mathrm{H}_{2} \mathrm{O}(10 / 1, \mathrm{v} / \mathrm{v}, 11.7 \mathrm{ml})$ was added $\mathrm{PPh}_{3}$ (431 mg, $1.64 \mathrm{mmol}$ ) under Ar atmosphere at room temperature. After the mixture was stirred for $15 \mathrm{~h}$ at $40{ }^{\circ} \mathrm{C}$, the mixture was quenched by addition of $\mathrm{H}_{2} \mathrm{O}(5 \mathrm{ml})$. The resulting mixture was extracted with $\mathrm{Et}_{2} \mathrm{O}$ $(5 \mathrm{ml} \times 3)$. The combined organic layer was washed with brine $(5 \mathrm{ml})$, dried over anhydrous $\mathrm{Na}_{2} \mathrm{SO}_{4}$, and concentrated in vacuo. Purification of the residue by silica-gel column chromatography $\left(\mathrm{CHCl}_{3} / \mathrm{MeOH}=10 / 1-5 / 1,1 \% \mathrm{NH}_{3}\right.$ aq. $)$ gave 8 (101 mg, $806 \mu \mathrm{mol}, 98 \%$ yield). Pale yellow syrup; $R_{f} 0.23\left(5 / 1 \mathrm{CHCl}_{3} /\right.$ $\mathrm{MeOH}) ;[\alpha]_{\mathrm{D}}^{24}+8.21^{\circ}\left(c 0.84, \mathrm{CHCl}_{3}\right) ;{ }^{1} \mathrm{H}-\mathrm{NMR}\left(\mathrm{CDCl}_{3}, \mathrm{TMS}\right) \delta 6.39(1 \mathrm{H}$, dd, $J=10.6,17.5 \mathrm{~Hz}), 5.51(1 \mathrm{H}, \mathrm{t}, J=7.5 \mathrm{~Hz}), 5.11(1 \mathrm{H}, \mathrm{d}, J=17.5 \mathrm{~Hz}), 4.95$ $(1 \mathrm{H}, \mathrm{d}, J=10.6 \mathrm{~Hz}), 2.99(1 \mathrm{H}, \mathrm{m}), 2.19(2 \mathrm{H}, \mathrm{dd}, J=7.2,7.5 \mathrm{~Hz}), 1.74(3 \mathrm{H}, \mathrm{s})$, $1.42(2 \mathrm{H}, \mathrm{s}), 1.09(3 \mathrm{H}, \mathrm{d}, J=6.3 \mathrm{~Hz}) ;{ }^{13} \mathrm{C}-\mathrm{NMR}\left(\mathrm{CDCl}_{3}\right) \delta 141.5,136.0,129.9$, 111.1, 47.4, 38.9, 23.7, 12.0; HRMS (ESI-TOF) $\mathrm{m} / \mathrm{z} 126.1283$ (126.1283 calcd for $\left.\mathrm{C}_{8} \mathrm{H}_{16} \mathrm{~N},[\mathrm{M}+\mathrm{H}]^{+}\right)$.

\section{$(2 Z, 4 \mathrm{E}, 6 E, 8 E, 10 R, 11 S, 12 Z)-1-((2 E, 4 E, 6 E)-5-M e t h y l h e p t a-4,6-$} diene)amide-10,11-bis(triethylsilyloxy)-13-iodo-2-methoxy-4,10,16trimethyltrideca-2,4,6,8,12-pentaene (18)

To a solution of $3(114 \mathrm{mg}, 176 \mu \mathrm{mol})$ in $\mathrm{CH}_{2} \mathrm{Cl}_{2}(1.50 \mathrm{ml})$ were added DMAP $(43.0 \mathrm{mg}, 352 \mu \mathrm{mol})$ and $\mathrm{EDC}(67.4 \mathrm{mg}, 352 \mu \mathrm{mol})$ under $\mathrm{Ar}$ atmosphere at $0{ }^{\circ} \mathrm{C}$. After the mixture was stirred for $20 \mathrm{~min}$ at the temperature, a solution of $8(88.1 \mathrm{mg}, 704 \mu \mathrm{mol})$ in $\mathrm{CH}_{2} \mathrm{Cl}_{2}(2.00 \mathrm{ml})$ was added and stirring was continued for another $13 \mathrm{~h}$ at room temperature. The reaction was quenched by addition of $\mathrm{H}_{2} \mathrm{O}(2 \mathrm{ml})$. The resulting mixture was extracted with EtOAc $(2 \mathrm{ml} \times 3)$. The combined organic layer was washed with brine $(2 \mathrm{ml})$, dried over anhydrous $\mathrm{Na}_{2} \mathrm{SO}_{4}$, and concentrated in vacuo. Purification of the residue by silica-gel column chromatography (hexane/EtOAc $=10 / 1$ to $4 / 1,1 \% \mathrm{Et}_{3} \mathrm{~N}$ ) gave 18 ( $115 \mathrm{mg}, 152 \mu \mathrm{mol}, 94 \%$ yield). Pale yellow syrup; $R_{f} 0.30$ (5/1 hexane/ EtOAc); $[\alpha]_{\mathrm{D}}^{26}+23.7^{\circ}\left(\right.$ c $\left.0.89, \mathrm{CHCl}_{3}\right) ;{ }^{1} \mathrm{H}-\mathrm{NMR}\left(\mathrm{CDCl}_{3}, \mathrm{TMS}\right) \delta 6.67(1 \mathrm{H}, \mathrm{s})$, $6.51(1 \mathrm{H}, \mathrm{dd}, J=11.5,14.0 \mathrm{~Hz}), 6.40(1 \mathrm{H}, \mathrm{d}, J=11.5 \mathrm{~Hz}), 6.38(1 \mathrm{H}, \mathrm{dd}$, $J=10.9,17.5 \mathrm{~Hz}), 6.33(1 \mathrm{H}, \mathrm{dd}, J=10.9,14 \mathrm{~Hz}), 6.32(1 \mathrm{H}, \mathrm{d}, J=7.7 \mathrm{~Hz}), 6.24$ $(1 \mathrm{H}, \mathrm{dd}, J=10.9,15.2 \mathrm{~Hz}), 6.15(1 \mathrm{H}, \mathrm{dd}, J=7.7,8.3 \mathrm{~Hz}), 5.86(1 \mathrm{H}, \mathrm{d}, J=15.2$ $\mathrm{Hz}), 5.50(1 \mathrm{H}, \mathrm{t}, J=7.5 \mathrm{~Hz}), 5.12(1 \mathrm{H}, \mathrm{d}, J=17.5 \mathrm{~Hz}), 4.97(1 \mathrm{H}, \mathrm{d}, J=10.9$ $\mathrm{Hz}), 4.14(1 \mathrm{H}, \mathrm{m}), 4.12(1 \mathrm{H}, \mathrm{d}, J=8.3 \mathrm{~Hz}), 3.57(3 \mathrm{H}, \mathrm{s}), 2.38(2 \mathrm{H}, \mathrm{dd}, J=6.9$, $7.5 \mathrm{~Hz}), 2.09(3 \mathrm{H}, \mathrm{s}), 1.77(3 \mathrm{H}, \mathrm{s}), 1.35(3 \mathrm{H}, \mathrm{s}), 1.20(3 \mathrm{H}, \mathrm{d}, J=6.6 \mathrm{~Hz}), 0.93$ $(18 \mathrm{H}, \mathrm{m}), 0.59(12 \mathrm{H}, \mathrm{m}) ;{ }^{13} \mathrm{C}-\mathrm{NMR}\left(\mathrm{CDCl}_{3}\right) \delta 163.7,147.3,141.3 \times 2,140.8$, $136.7,135.9,135.4,131.8,129.6,128.4,128.1,124.8,111.6,83.6,81.3,78.3$, $61.1,45.5,35.2,22.9,20.6,15.0,12.1,7.3,7.0,6.8,5.2$; HRMS (ESI-TOF) $\mathrm{m} / \mathrm{z}$ 756.3340 (756.3340 calcd for $\mathrm{C}_{36} \mathrm{H}_{63} \mathrm{NO}_{4} \mathrm{Si}_{2} \mathrm{I},[\mathrm{M}+\mathrm{H}]^{+}$).

\section{$(2 Z, 4 E, 6 E, 8 E, 10 R, 11 S, 12 Z, 14 E, 16 E, 18 E)-1-((2 E, 4 E, 6 E)-5-$} Methylhepta-4,6-diene)amide-10,11-bis(triethylsilyloxy)-13-iodo-2methoxy-4,10,16-trimethylnonadeca-2,4,6,8,12,14,16-heptaene (19) To a solution of $18(25.8 \mathrm{mg}, 34.1 \mu \mathrm{mol})$ and $7(78.5 \mathrm{mg}, 205 \mu \mathrm{mol})$ in dry THF/DMF $(1 / 1, \mathrm{v} / \mathrm{v}, 683 \mu \mathrm{l})$ were added $\mathrm{LiCl}(11.6 \mathrm{mg}, 273 \mu \mathrm{mol}), \mathrm{CuCl}$ $(20.3 \mathrm{mg}, 205 \mu \mathrm{mol})$ and $\mathrm{Pd}_{2}(\mathrm{dba})_{3}(6.3 \mathrm{mg}, 6.83 \mu \mathrm{mol})$ under $\mathrm{Ar}$ atmosphere at room temperature. After the mixture was stirred for $4 \mathrm{~h}$, the reaction was quenched by addition of saturated $\mathrm{NaHCO}_{3}$ aq. $(1 \mathrm{ml})$. The resulting mixture was extracted with hexane/EtOAc $(1 / 1, \mathrm{v} / \mathrm{v}, 1 \mathrm{ml} \times 3)$. The combined organic layer was washed with brine $(5 \mathrm{ml})$, dried over anhydrous $\mathrm{Na}_{2} \mathrm{SO}_{4}$, and concentrated in vacuo. Purification of the residue by silica-gel column chromatography (hexane/EtOAc $=10 / 1$ to $\left.8 / 1,1 \% \mathrm{Et}_{3} \mathrm{~N}\right)$ gave $19(16.5 \mathrm{mg}$, $22.8 \mu \mathrm{mol}, 71 \%$ yield). Pale yellow syrup: $R_{f} 0.31$ (5/1 hexane/EtOAc); $[\alpha]_{D}^{26}$ $-86.8^{\circ}\left(c 0.98, \mathrm{CHCl}_{3}\right) ;{ }^{1} \mathrm{H}-\mathrm{NMR}\left(\mathrm{CDCl}_{3}\right.$, TMS $) \delta 6.72(1 \mathrm{H}, \mathrm{ddd}, J=10.0$, $11.2,16.6 \mathrm{~Hz}), 6.56(1 \mathrm{H}, \mathrm{dd}, J=11.2,15.2 \mathrm{~Hz}), 6.51(1 \mathrm{H}, \mathrm{dd}, J=11.5$, $14.1 \mathrm{~Hz}), 6.41(1 \mathrm{H}, \mathrm{d}, J=11.5 \mathrm{~Hz}), 6.37(1 \mathrm{H}, \mathrm{dd}, J=11.2,17.5 \mathrm{~Hz}), 6.32$ $(1 \mathrm{H}, \mathrm{dd}, J=11.8,14.1 \mathrm{~Hz}), 6.26(1 \mathrm{H}, \mathrm{d}, J=15.2 \mathrm{~Hz}), 6.25(1 \mathrm{H}, \mathrm{dd}, J=11.8$ $14.9 \mathrm{~Hz}), 6.12(1 \mathrm{H}, \mathrm{d}, J=11.2 \mathrm{~Hz}), 6.11(1 \mathrm{H}, \mathrm{dd}, J=10.3,11.2 \mathrm{~Hz}), 5.91(1 \mathrm{H}$, d, $J=14.9 \mathrm{~Hz}), 5.50(1 \mathrm{H}, \mathrm{t}, J=7.2 \mathrm{~Hz}), 5.37(1 \mathrm{H}, \mathrm{dd}, J=9.2,10.3 \mathrm{~Hz}), 5.26$ $(1 \mathrm{H}, \mathrm{d}, J=16.6 \mathrm{~Hz}), 5.15(1 \mathrm{H}, \mathrm{d}, J=10.0 \mathrm{~Hz}), 5.12(1 \mathrm{H}, \mathrm{d}, J=17.5 \mathrm{~Hz}), 4.97$ $(1 \mathrm{H}, \mathrm{d}, J=11.2 \mathrm{~Hz}), 4.32(1 \mathrm{H}, \mathrm{d}, J=9.2 \mathrm{~Hz}), 4.14(1 \mathrm{H}, \mathrm{m}), 3.57(3 \mathrm{H}, \mathrm{s}), 2.38$ $(2 \mathrm{H}, \mathrm{dd}, J=6.9,7.2 \mathrm{~Hz}), 2.09(3 \mathrm{H}, \mathrm{s}), 1.90(3 \mathrm{H}, \mathrm{s}), 1.77(3 \mathrm{H}, \mathrm{s}), 1.31(3 \mathrm{H}, \mathrm{s})$ $1.20(3 \mathrm{H}, \mathrm{d}, J=6.6 \mathrm{~Hz}), 0.91(18 \mathrm{H}, \mathrm{m}), 0.59(12 \mathrm{H}, \mathrm{m}) ;{ }^{13} \mathrm{C}-\mathrm{NMR}\left(\mathrm{CDCl}_{3}\right) \delta$ $163.7,147.3,142.1,141.3,138.3,136.7,136.0,135.9,135.6,133.3,132.3,131.7$, $131.6,130.8,129.2,128.2,128.1 \times 2,125.0,124.8,117.8,111.6,61.1,45.5,35.2$, 
21.8, 20.6, 15.0, 12.8, 12.1, 7.4, 7.3, 7.0, 6.8, 5.1; HRMS (ESI-TOF) $\mathrm{m} / \mathrm{z}$ 722.4979 (722.5000 calcd for $\left.\mathrm{C}_{43} \mathrm{H}_{72} \mathrm{NO}_{4} \mathrm{Si}_{2},[\mathrm{M}+\mathrm{H}]^{+}\right)$.

\section{Incednam (1)}

To a stirred solution of $19(16.6 \mathrm{mg}, 23.0 \mu \mathrm{mol})$ in deaerated $\mathrm{CH}_{2} \mathrm{Cl}_{2}(12.0 \mathrm{ml})$ was added molecular sieves $3 \mathrm{~A}(16.6 \mathrm{mg})$, p-methoxyphenol $(5.7 \mathrm{mg}$, $46.0 \mu \mathrm{mol})$ and Grela catalyst $21(6.2 \mathrm{mg}, 9.20 \mu \mathrm{mol})$ at room temperature. After stirring at the temperature for $6.5 \mathrm{~h}$, the solution was passed through silica-gel column chromatography $\left(\mathrm{CHCl}_{3}, 1 \% \mathrm{Et}_{3} \mathrm{~N}\right)$, and concentrated in vacuo. The crude product $(3.9 \mathrm{mg})$ was used for the next reaction without further purification. To a solution of crude product $(3.9 \mathrm{mg})$ in dry THF $(562 \mu \mathrm{l})$ were added the mixture of $1.0 \mathrm{M}$ TBAF in THF $(33.7 \mu \mathrm{l}, 33.7 \mu \mathrm{mol})$ and $\mathrm{AcOH}(1.6 \mu \mathrm{l}, 28.1 \mu \mathrm{mol})$ under Ar atmosphere. The reaction mixture was stirred for $16 \mathrm{~h}$ at room temperature, and then the mixture of $1.0 \mathrm{M}$ TBAF in THF $(67.4 \mu \mathrm{l}, 67.4 \mu \mathrm{mol})$ and $\mathrm{AcOH}(3.2 \mu \mathrm{l}, 56.2 \mu \mathrm{mol})$ was added at the same temperature. After the mixture was stirred for $8 \mathrm{~h}$ at the temperature, the reaction was quenched by addition of saturated $\mathrm{NaHCO}_{3}$ aq. $(2 \mathrm{ml})$. The resulting mixture was extracted with EtOAc $(2 \mathrm{ml} \times 3)$. The combined organic layer was washed with brine $(2 \mathrm{ml})$, dried over anhydrous $\mathrm{Na}_{2} \mathrm{SO}_{4}$, and concentrated in vacuo. Purification of the residue by silica-gel column chromatography $\left(\mathrm{CHCl}_{3} / \mathrm{MeOH}=40 / 1,1 \% \mathrm{Et}_{3} \mathrm{~N}\right)$ gave $1(1.8 \mathrm{mg}, 3.9 \mu \mathrm{mol}$, $17 \%$ yield in two steps). Data for an analytical sample of the synthetic incednam (1) obtained by ${ }^{1} \mathrm{H}-\mathrm{NMR}$, HRMS (ESI-TOF) and optical rotation matched those obtained for an authentic sample and a sample from the 1st generation synthesis. ${ }^{1,5}$ Pale yellow powder; $R_{f} 0.46\left(10 / 1 \mathrm{CHCl}_{3} / \mathrm{MeOH}\right) ;[\alpha]_{D}^{26}$ $-1469.4^{\circ}$ (c $\left.0.10, \mathrm{CHCl}_{3}\right)$, lit. ${ }^{1}[\alpha]_{\mathrm{D}}^{20}-1616.7^{\circ}$ (c $\left.0.1, \mathrm{CHCl}_{3}\right) ;{ }^{1} \mathrm{H}-\mathrm{NMR}$ $\left(\mathrm{CDCl}_{3}: \mathrm{CD}_{3} \mathrm{OD}=1: 1, \mathrm{TMS}\right) \delta 6.73(1 \mathrm{H}, \mathrm{d}, J=10.6 \mathrm{~Hz}) 6.43(1 \mathrm{H}, \mathrm{dd}, J=11.8$, $14.4 \mathrm{~Hz}), 6.21(1 \mathrm{H}, \mathrm{m}), 6.21(1 \mathrm{H}, \mathrm{m}), 6.19(1 \mathrm{H}, \mathrm{dd}, J=10.9,15.8 \mathrm{~Hz}), 6.18$ $(1 \mathrm{H}, \mathrm{d}, J=15.4 \mathrm{~Hz}), 6.15(2 \mathrm{H}, \mathrm{m}), 6.10(1 \mathrm{H}, \mathrm{m}), 6.05(1 \mathrm{H}, \mathrm{d}, J=10.6 \mathrm{~Hz})$, $6.02(1 \mathrm{H}, \mathrm{dd}, J=10.9,14.4 \mathrm{~Hz}), 5.95(1 \mathrm{H}, \mathrm{d}, J=11.5 \mathrm{~Hz}), 5.65(1 \mathrm{H}, \mathrm{d}$, $J=15.8 \mathrm{~Hz}), 5.45(1 \mathrm{H}, \mathrm{t}, J=9.2), 5.45(1 \mathrm{H}, \mathrm{m}), 4.22(1 \mathrm{H}, \mathrm{d}, J=8.6 \mathrm{~Hz}), 4.17$ $(1 \mathrm{H}, \mathrm{m}), 3.58(3 \mathrm{H}, \mathrm{s}), 2.28(1 \mathrm{H}, \mathrm{m}), 2.32(1 \mathrm{H}, \mathrm{m}), 2.09(3 \mathrm{H}, \mathrm{s}), 1.70(3 \mathrm{H}, \mathrm{s})$, $1.66(3 \mathrm{H}, \quad \mathrm{s}), \quad 1.49(3 \mathrm{H}, \mathrm{s}), \quad 1.32(3 \mathrm{H}, \quad \mathrm{d}, \quad J=6.7 \mathrm{~Hz} \mathrm{~Hz}) ;{ }^{13} \mathrm{C}-\mathrm{NMR}$ $\left(\mathrm{CDCl}_{3}: \mathrm{CD}_{3} \mathrm{OD}=1: 1\right) \delta 167.0,147.4,143.4,138.0,137.8 \times 3,137.4,135.5$, $132.4 \times 2,130.7,130.6,130.0,129.1,128.1,127.1,125.4,124.6,76.2,76.0,61.3$, $47.2,38.5,22.8,20.9,14.3,13.0,12.8$; HRMS (ESI-TOF) $\mathrm{m} / \mathrm{z} 466.2948$ (466.2957 calcd for $\mathrm{C}_{29} \mathrm{H}_{40} \mathrm{NO}_{4},[\mathrm{M}+\mathrm{H}]^{+}$).
1 Futamura, Y. et al. Discovery of incednine as a potent modulator of the anti-apoptotic function of Bcl-xL from microbial origin. J. Am. Chem. Soc. 130, 1822-1823 (2008).

2 Tsujimoto, Y., Finger, L. R., Yunis, J., Nowell, P. C. \& Croce, C. M. Cloning of the chromosome breakpoint of neoplastic B cells with the $\mathrm{t}(14 ; 18)$ chromosome translocation. Science 226, 1097-1099 (1984).

3 Reed, J. C., Cuddy, M., Slabiak, T., Croce, C. M. \& Nowell, P. C. Oncogenic potential of bcl-2 demonstrated by gene transfer. Nature 336, 259-261 (1988).

4 Gross, A., McDonnell, J. M. \& Korsmeyer, S. J. BCL-2 family members and the mitochondria in apoptosis. Genes Dev. 13, 1899-1911 (1999).

5 Ohtani, T. et al. Total synthesis of incednam, the aglycon of incednine. Org. Lett. 12, 5068-5071 (2010).

6 Grubbs, R. H. Olefin metathesis. Tetrahedron 60, 7117-7140 (2004).

7 Gradillas, A. \& Pérez-Castells, J. Macrocyclization by ring-closing metathesis in the total synthesis of natural products: reaction conditions and limitations. Angew. Chem. Int. Ed. 45, 6086-6101 (2006).

8 Nicolaou, K. C., Bulger, P. G. \& Sarlah, D. Metathesis reactions in total synthesis. Angew. Chem. Int. Ed. 44, 4490-4527 (2005).

9 Betzer, J. -F., Delaloge, F., Muller, B., Pancrazi, A. \& Prunet, J. Radical hydrostannylation, $\mathrm{Pd}(0)$-catalyzed hydrostannylation, stannylcupration of propargyl alcohols and enynols: regio- and stereoselectivities. J. Org. Chem. 62, 7768-7780 (1997).

10 Michels, T. D., Rhee, J. U. \& Vanderwal, C. D. Synthesis of $\delta$-tributylstannyl- $\alpha, \beta, \gamma, \delta$ unsaturated aldehydes from pyridines. Org. Lett. 10, 4787-4790 (2008).

11 Staudinger, H. \& Meyer, J. New organic compounds of phosphorus. III. Phosphinemethylene derivatives and phosphinimines. Helv. Chim. Acta 2, 635-646 (1919).

12 Stuckwisch, C. G. Azomethine ylides, azomethine imines, and iminophosphoranes in organic syntheses. Synthesis 469-483 (1973).

13 Han, X., Stoltz, B. M. \& Corey, E. J. Cuprous chloride accelerated Stille reactions. A general and effective coupling system for sterically congested substrates and for enantioselective synthesis. J. Am. Chem. Soc. 121, 7600-7605 (1999).

14 Schwab, P., France, M. B., Ziller, J. W. \& Grubbs, R. H. A series of well-defined metathesis catalysts-synthesis of $\left[\mathrm{RuCl}_{2}\left(: \mathrm{CHR}^{\prime}\right)\left(\mathrm{PR}_{3}\right)_{2}\right]$ and its reactions. Angew. Chem. Int. Ed. 34, 2039-2041 (1995).

15 Scholl, M., Trnka, T. M., Morgan, J. P. \& Grubbs, R. H. Increased ring closing metathesis activity of ruthenium-based olefin metathesis catalysts coordinated with imidazolin-2-ylidene ligands. Tetrahedron Lett. 40, 2247-2250 (1999).

16 Kingsbury, J. S., Harrity, J. P. A., Bonitatebus, P. J. Jr \& Hoveyda, A. H. A recyclable Ru-based metathesis catalyst. J. Am. Chem. Soc 121, 791-799 (1999).

17 Garber, S. B., Kingsbury, J. S., Gray, B. L. \& Hoveyda, A. H. Efficient and recyclable monomeric and dendritic Ru-based metathesis catalysts. J. Am. Chem. Soc. 122, 8168-8179 (2000).

18 Grela, K., Harutyunyan, S. \& Michrowska, A. A highly efficient ruthenium catalyst for metathesis reactions. Angew. Chem. Int. Ed. 41, 4038-4040 (2002).

19 Bieniek, M., Michrowska, A., Gułajski, Ł. \& Grela, K. A practical larger scale preparation of second-generation Hoveyda-type catalysts. Organometallics 26, 1096-1099 (2007).

20 Ohtani, T., Sakai, S., Takada, A., Takahashi, D. \& Toshima, K. Efficient and stereoselective synthesis of the disaccharide fragment of incednine. Org. Lett. 13, 6126-6129 (2011). 